\title{
The Association Between Variants in PLA2R and HLA-DQA1 and Renal Outcomes in Patients With Primary Membranous Nephropathy in Western China
}

\author{
Shulei Fan \\ Nephrology \\ Qiuxia Wang \\ Nephrology \\ Amanda Y Wang \\ Nephrology \\ Ping Zhang \\ Nephrology \\ Xiang Zhong \\ Nephrology \\ Shasha Chen \\ Nephrology \\ Guisen Li \\ Nephrology \\ Li Wang \\ Nephrology \\ Wei Wang ( $\sim$ wwei958@163.com ) \\ Nephrology https://orcid.org/0000-0001-5664-7725
}

\section{Research article}

Keywords: Primary membranous nephropathy (PMN), M-type phospholipase A2 receptor (PLA2R), human leukocyte antigen complex class II HLA-DQa-chain 1 (HLA-DQA1), genotype, renal outcome

Posted Date: December 30th, 2020

DOl: https://doi.org/10.21203/rs.3.rs-136132/v1

License: (c) (1) This work is licensed under a Creative Commons Attribution 4.0 International License.

Read Full License 


\section{Abstract}

Background冈Both Genome-wide associations and our previous study have shown that single nucleotide polymorphisms (SNPs) of M-type phospholipase A2 receptor (PLA2R) and human leukocyte antigen complex class II HLA-DQa-chain 1 (HLA-DQA1) gene were identified to be associated with primary membranous nephropathy (PMN). However, whether these SNPs affect clinical manifestation and renal outcome for PMN patients is poorly defined. Here, we evaluated whether there is an association between these SNPs and clinical manifestations and renal outcomes of PMN in a western Chinese cohort.

Methods: Seven SNPs within PLA2R and one SNP in HLA-DQA1 were selected in our study. Clinical data from 314 patients with PMN were collected and the relationship between the genotype and phenotype was evaluated. A total of 186 patients had follow-up data. We assessed the treatment responses and renal outcomes between patients with these gene polymorphisms after a median follow-up of 18.6 months.

Results: Eight SNPs were not associated clinical manifestations of PMN patients ( $\mathrm{Pc}<0.05)$. rs3828323 T allele was marginally significantly associated with hypertension $(P=0.008, P C=0.064,0 \mathrm{R}=1.821)$. After treatment for $\mathrm{PMN}$, urea (OR=1.184, 95\% Cl:1.030-1.361, $P=0.017)$ was a risk factor for non-remission of the disease, eGFR (OR=0.984, 95\% Cl:0.970-0.998, $P=0.028)$ and albumin (OR=0.922, 95\% Cl:0.880-0.966, $P=0.001)$ were protective factors for non-remission of the disease. We also identified that PMN patients with CT/TT genotype for rs3828323 achieved higher cumulative survival rate than patients with CC genotype.

Conclusions $\mathbb{R}$ R3828323 may influence hypertension and renal outcome in patients with PMN. Further research is needed to explore the mechanism for this genotype-disease phenotype association.

\section{Background}

Primary membranous nephropathy (PMN) is one of the most common causes for nephrotic syndrome for adult. Its typical pathological features are diffuse thickening of glomerular capillary basement membrane and deposition of subepithelial immune complexes[1,2]. In recent years, its incidence is increasing. It is the second most common type of primary glomerulonephritis in China[3, 4].

PMN is a typical kidney disease caused by antigen-antibody reaction. Antigen-antibody binding forms immune complex, then activates complement system, resulting in injury of podocytes and glomerular basement membrane, and eventually leading to kidney injury $[5,6]$. The production of circulating PLA2R antibodies may be the main pathogenic mechanism of the disease[7]. The anti-PLA2R antibody has high specificity and good sensitivity. $70 \%$ to $80 \%$ of PMN patients are PLA2R positive. The higher the antibody titer is, the higher the risk of deterioration of renal function is $[8,9]$. It is reported that some clinical factors are related to the progress of the disease, including severe proteinuria, hypertension, and renal dysfunction at diagnosis[10, 11]. 
In Caucasian population, genome-wide association studies have confirmed the susceptibility of PLA2R and HLA-DQA1 gene with PMN[12]. Multiple loci in PLA2R and HLA-DQA1 were closely related to PMN in various ethnicities, but the results of different regions and ethnic groups were not entirely consistent [1218]. To verify the previous findings in Western China, in our previous study we selected eight SNPs reported in the literatures and found that two SNPs (rs2715918, rs4665143) within PLA2R, 1 SNP in HLADQA1 (rs2187668) were associated with primary membranous nephropathy[19]. The interactions of rs2715918, rs4665143 and rs2187668 were associated with an increased risk of the development of PMN by 10.61 -fold. Patients carrying risk alleles confer a predisposition to anti-PLA2R autoantibody generation[19]. In a Spanish study, Bullich et al found that PMN patients with two SNP risk alleles, rs2187668 and rs4664308, had better response to immunosuppressive therapy and slower progression of chronic kidney disease[17]. Wang et al found that PMN Patients with HLA-DRB1*1502 had worse kidney outcomes in Han Chinese[20].

To evaluate whether these SNPs are associated with clinical manifestations and renal outcomes of PMN patients, clinical data from 314 patients with PMN were collected and the relationship between the genotype and phenotype was evaluated. We performed a retrospective cohort study of 186 patients who had follow-up data to assess the relationship between genetic polymorphisms and renal outcome.

\section{Methods}

\section{Patients}

From January 2010 to December 2016, patients with biopsy-proven PMN in Sichuan Provincial People's Hospital were recruited. Among them, 314 patients who had complete clinical baseline data in our Renal Treatment System (RTS) database were included in the study. All the patients came from Western Han ethnicity. In addition, the data on complete follow-up more than 3months were available and recorded in 186 patients. Patients with membranous nephropathy due to secondary causes, such as systemic lupus erythematosus, cancer, hepatitis B virus infection and drug were excluded.

The study was approved by the Ethics Committee of the Sichuan Provincial People's Hospital (Chengdu, China), and all the patients signed informed consents to participate in this study.

Genotyping of PLA2Rand HLA-DQA1

The methods for selection of candidate SNPs of PLA2Rand HLA-DQA1 and genotyping were described in the previous study[19]. SNPs of the candidate genes were obtained from previously published polymorphisms associated with PMN.

\section{Genotype-phenotype correlation studies}

Baseline clinical data were collected from all patients at diagnosis. PMN patients were divided into several subgroups based on the following parameters: 24 hours urinary protein excretion (24h-u-pro $\geq 3.5 \mathrm{~g} / \mathrm{d}$ or $<3.5 \mathrm{~g} / \mathrm{d}$ ), renal function (eGFR $\leq 60$ or $>60 \mathrm{~mL} / \mathrm{min} / 1.73 \mathrm{~m}^{2}$ ), and blood pressure ( $\geq 140 / 90 \mathrm{mmHg}$ or 
$<140 / 90 \mathrm{mmHg}$ ). The eGFR calculation was using the CKD-EPI equation[32]. The association of alleles frequencies, genotype frequencies and different genetic models with clinical phenotype (24-h-pro, eGFR and blood pressure) in PMN patients was analyzed.

\section{Detection PLA2R antibody in serum}

120 PMN patients had anti-PLA2R antibodies detected to analyze the relationship between anti-PLA2R positivity and treatment response, as well as renal outcomes. The methods for measuring PLA2R antibody in serum were described in the previous study[19].

\section{Definition}

ESRD was defined as eGFR $<15 \mathrm{~mL} / \mathrm{min} / 1.73 \mathrm{~m}^{2}$, receiving dialysis therapy for more than 3 months or transplantation[33]. The primary outcome was renal progression, defined as a composite of ESRD, a reduction in eGFR by $>30 \%$ from the baseline and doubling serum creatinine. Spontaneous remission (SR) included complete remission and partial remission. Complete remission (CR) was defined as 24 hours urinary protein excretion $<500 \mathrm{mg}$ per day with normal serum creatinine and serum albumin ( $\geq 35 \mathrm{~g} / \mathrm{L})$. Partial remission (PR) was defined as 24 hours urinary protein excretion of $0.5-2 \mathrm{~g}$ per day or $<50 \%$ of baseline with normal serum creatinine and serum albumin level [24]. Patients who did not have complete or partial remission were called non- spontaneous remission. According our previous study, the PLA2R low-risk was defined as individuals with GG in rs2715918 and GG in rs4665143 within PLA2R. The rest were the PLA2R high-risk group. The HLA-DQA1 low-risk was defined as individuals with GG in rs2187668 within HLA-DQA1. The rest were the HLA-DQA1 high-risk group[19].

\section{Statistical analysis}

Continuous variables were presented as mean and standard deviation quartile spacing standard deviation (SD)Categorical variables were presented as median with interquartile ranges (IQR). Chi-square test was used to compare the classified variables between the two groups. Categorical variables were presented by proportions. Comparisons of continuous variables using t-test or one-way ANOVA. Hardy-Weinberg equilibrium (HWE) was determined by chi-square test. Logistic regression analysis was used to test three kinds of models of inheritance. Bonferroni adjustment was performed to correct $\mathrm{P}$ values $(\mathrm{Pc})$. The association between gene mutation and kidney survival was analyzed by Kaplan-Meier.

\section{Results}

\section{Baseline characteristics of PMN patients}

The baseline characteristics of 314 (166 male and 148 female) PMN patients were described in Table 1. All those who participated in the study lived in Sichuan province for several generations and belonged to the Han ethnicity. The mean age at the time of renal biopsy was $49.1 \pm 13.5$ years old, and the mean eGFR was $101.33 \pm 22.67 \mathrm{ml} / \mathrm{min}$ per $1.73 \mathrm{~m}^{2}$. The median baseline proteinuria was 4.03 (range,2.34-6.20) g/24 h. 


\section{Genotype-phenotype correlations}

Eight SNPs were successfully genotyped. The details of SNPs were shown in our pervious study[19]. All of them conformed to the Hardy-Weinberg equilibrium (HWE). We determined the association of alleles, genotype frequencies and different genetic models of candidate eights SNPs with phenotype (24h-u-pro, eGFR, blood pressure, respectively) in 314 PMN patients (Supplementary Table S1, Supplementary Table S2, Supplementary Table S3, respectively). The result revealed significant differences in alleles, genotype distributions, dominant, recessive, additive model of rs3828323 with hypertension $(P=0.008,0.029,0.037$, $0.034,0.012$, respectively). After Bonferroni adjustment, there was no statistical significance in the above variables $(P=0.064,0.232,0.296,0.272,0.096$, respectively). There was no association of alleles frequencies, genotype frequencies and different genetic models of rs3828323 with 24-h-pro or eGFR. No association was found between other SNPs and clinical phenotypes (24h-u-pro, eGFR, blood pressure). These results indicated the potential effects of rs3828323 on hypertension.

\section{Follow-up data and treatment response}

Among all the 314 individuals, 186 patients had complete follow-up data. Their baseline and follow-up characteristics were described in table 2. Forty-one (22.0\%) patients reached the composite outcome during a median follow-up time of 18.6 (range, 6.7-45.5) months. The numbers of corticosteroid monotherapy, immunosuppression (Steroid + cyclophosphamide, Calcineurin inhibitor with or without steroid) and non-immunosuppression (with angiotensin-converting enzyme inhibitors or angiotensin II receptor blockers) were $29(15.6 \%), 112(60.2 \%), 45(24.2 \%)$, respectively. The result also showed that among 112 patients receiving immunosuppressive therapy, the numbers of complete remission, partial remission and non-spontaneous remission were 37(33.0\%), 21(18.8\%), 54(48.2\%), respectively. Among 45 patients who did not receive immunosuppressive therapy, the numbers of complete remission, partial remission and non-spontaneous remission were 14(31.1\%), 13(28.9\%), 18(40.0\%), respectively (Table 2).

\section{Risk factors for no remission after treatment in PMN}

According to the logistic regression analysis, we found urea (OR=1.184, 95\% Cl:1.030-1.361, $P=0.017)$ was a risk factor for non-remission of the disease, eGFR (OR=0.984, 95\% Cl:0.970-0.998, $P=0.028)$ and albumin $(\mathrm{OR}=0.922,95 \% \mathrm{Cl}: 0.880-0.966, P=0.001)$ were protective factors for non-remission of the disease (Table $3)$.

\section{Genetic Variants and Immunosuppressive Therapy Response}

Our previous studies showed interaction of rs 2715918 GA/AA, rs4665143 GA/AA and rs2187668 GA/AA could significantly increase the risk for the development of PMN by 10.61-fold [19]. Therefore, in this study, we still choose these 3 SNPs and detected the association between genetic variants and response to immunosuppressive therapy. Genotypes under a dominant mode were considered the non-risk genotypes for PMN susceptibility and used as a reference. In univariate and multivariate logistic regression analysis, we found no statistical significance in response to immunosuppressive therapy between the reference and the genotypes carried risk alleles (Table 4). 


\section{Genetic variants and decline in renal function}

Forty-one (22.0\%) patients reached the composite outcome during a median follow-up time of 18.6 (range, 6.7-45.5) months. Kaplan-Meier analysis showed that for rs3828323, patients carrying CT/TT genotype achieved superior outcomes than CC genotype ( $P=0.003$, Fig. 1$)$. No associations were found with other SNPs.

\section{Association between PLA2R/HLADQA1 Genotype and Anti-PLA2R Antibody}

Our study included 314 PMN patients, of which 120 patients had anti-PLA2R antibodies in plasma tested. According to our previous study, we found that patients with low-risk SNPs had lower anti-PLA2R antibody positive rate than patients with high-risk SNPs[19]. Furthermore, we found there was no statistically significant difference in baseline characteristics between anti-PLA2R antibody positive group and antiPLA2R antibody negative group (Table 5). Table 6 listed baseline characteristics in different combination groups. Both PLA2R and HLA-DQA1 high-risk group had the highest anti-PLA2R antibody positive rate (Table 6).

\section{Discussion}

In our retrospective study including 314 PMN patients, 8 SNPs of 2 genes (PLA2R and HLA-DQA1) were selected to analyze the association between SNPs and clinical phenotype in PMN patients. Rs3828323 T allele was marginally significantly associated with hypertension $(P=0.008, P c=0.064, \mathrm{OR}=1.821)$. Among 186 patients who had complete follow-up data, forty-one (22.0\%) patients reached the composite outcome during a median follow-up time of 18.6 (range, 6.7-45.5) months. The further study showed that patients carrying CT/TT genotype achieved better renal survival than patients carrying CC for rs3828323. The selected other candidate SNPs showed no association with clinical phenotype and renal outcomes in PMN patients.

Some of our results are consistent with the previous research. Liu et al found that rs35771982 and rs6757188 had no significant correlation with different clinical manifestations of PMN in a retrospective study and they were not independent risk factors for disease progression[14]. Kim et al identified that rs35771982 and rs3828323 were not associated with the prognosis of PMN patients[13]. However, in the current study, we found that patients with one or two T alleles (CT/TT) of rs382823 had higher kidney survival than those without T allele (CC).

The natural course of PMN is diverse, with about one third of patients able to achieve spontaneous remission, about one third of patients with persistent proteinuria but long-term stable renal function, and another third of patients progressing slowly to end stage renal disease (ESRD)[10, 21, 22]. In our cohort, the median follow-up time of patients was 18.6 (range, 6.7-45.5) months. 55.4\% ( $n=103)$ of them achieved spontaneous remission. In our data, urea (OR=1.184, 95\% Cl:1.030-1.361, $P=0.017)$ was a risk factor, eGFR $(\mathrm{OR}=0.984,95 \% \mathrm{Cl}: 0.970-0.998, P=0.028)$ and albumin (OR=0.922, 95\% $\mathrm{Cl}: 0.880-0.966, P=0.001)$ were 
protective factors for non-remission of the disease. This finding is consistent with the previous literature where female was shown as a predictor of SR[10,23].

Next, we compared genetic variants in terms of response to immunosuppressive therapy and renal outcomes. We found no statistically significant difference in response to immunosuppressive therapy. We also found that three risk genes for PMN development (A allele of rs2715918, A allele of rs4665143 and A allele of rs2187668) were not associated with renal outcomes and did not predict response to immunosuppressive therapy. However, we found that rs3828323 patients with one or two T alleles (CT/TT) had higher kidney survival than those without T allele (CC). In our previous study, rs3828323 was identified as a protective allele, indicating that a protective allele can not only reduce the susceptibility of PMN, but also serve as a predictor for real outcomes. Liu et al found that two loci rs35771982and 6757188 were associated with low remission rate after immunosuppressive therapy[14]. Bullich et al revealed that patients with both rs 2187668 AA/AG and rs4664308 AA had better therapeutic effect with immunosuppressive agents, and slower progression of renal disease[17]. Our results are not consistent with the previous research. There are several potential explanations. PMN is a complex disease with both genetic and environmental factors contributing to the progress of the disease. Genetic heterogeneity among different races, or environmental factors, can influence disease progression. Furthermore, various clinical conditions of PMN patients may also have an impact on the disease progression. Further studies with a large sample size and longer duration of follow up is needed to explore the impact of SNP on treatment.

Nonsynonymous SNP rs3828323 C>T within PLA2R (Gly1106Ser) is located in a coding region of exon 24[16]. Previous literature and our study have confirmed that it is related to PMN susceptibility[16, 19, 2426]. The SNP mutation sites of PLA2R can be found in both coding region or non-coding region, especially in intron or exon boundary region[16]. Any SNP may lead to different transcriptome lengths, which may lead to changes in protein function. A longer cut segment will form a stable transmembrane PLA2R protein, while a shorter cut segment will form a soluble enhanced PLA2R protein[27]. In conclusion, the SNP mutation of PLA2R gene may lead to the conformational change of PLA2R, and ultimately lead to the activation of PLA2R antibody, affecting the susceptibility and progress of the disease[24]. SNP mutation of HLA-DQA1 is more dangerous to PMN than PLA2R. There is a combined effect between these two mutations. Some researchers believed that HLA-DQA1 may be the dominant factor in the occurrence of PMN[12]. Its effect on the PMN autoantibody production may not only be through PLA2R pathway, but also through other ways[12]. In short, it seemed that the gene mutation of immunological immune system proteins (such as HLA-DQA1) is closely related to the gene mutation of glomerular component proteins (such as PLA2R), although the causal relationship between these two genes mutation is not yet clear. The direct relationship has not yet been established, but the whole mechanism is probably due to a complex system composed of trigger factors (immune system/HLA-DQA1), transduction pathway (PLA2R antibody) and target (glomerular antigen) related to[12] . Further researches on the occurrence and progress of SNP and PMN is needed. 
Patients with the low-risk alleles of PLA2R and HLA-DQA1 had the lowest positive rate for anti-PLA2R antibody (41.6\%). In contrast, patients with the high-risk alleles of PLA2R and HLA-DQA1 had the highest positive rate for anti-PLA2R antibody (87.8\%). In all PMN patients who were tested for anti-PLA2R antibodies, treatment responses were seen in 71 patients. Among them, 57 patients had positive antiPLA2R antibodies, of which 34 patients (59.6\%) achieved spontaneous remission. Among 14 patients with negative anti-PLA2R antibodies, only 5 patients (35.7\%) achieved spontaneous remission. Serum antiPLA2R antibodies can be used in clinical diagnosis of PMN, especially in elderly patients with nephrotic syndrome or those who are not suitable for renal biopsy[28]. Anti-PLA2R antibody has high specificity and sensitivity. High titer of anti-PLA2R antibody indicates high risk of deterioration of renal function, low possibility of clinical remission, high recurrence rate and high risk of recurrence after renal transplant $[8,29$, 30]. However, current literature reported the association between anti-PLA2R antibody and disease prognosis with inconsistent results[11, 24,31]. suggesting that further higher quality research is needed in the future.

The major limitation of our study was the relatively short duration of follow up and small sample size. We were unable to fully explore the association between anti-PLA2R autoantibodies and renal disease progression due to the retrospective nature of this study.

\section{Conclusion}

In conclusion, to our knowledge, this is the first study identified that patients with CT/TT genotype achieved higher cumulative survival rate than patients with CC genotype for rs3828323. Our study also showed that rs3828323 T allele was marginally significantly associated with hypertension. Urea was a risk factor, and eGFR and albumin were protective factors for non-remission after treatment for PMN.

\section{Abbreviations}

CR: complete remission

ESRD: end stage renal disease

HLA-DQA1: human leukocyte antigen complex class II HLA-DQa-chain 1

HWE: Hardy-Weinberg equilibrium

PLA2R: M-type phospholipase A2 receptor

PMN: primary membranous nephropathy

PR: partial remission

SNPs: single nucleotide polymorphisms

SR: spontaneous remission 


\section{Declarations}

\section{Ethics approval and consent to participate}

The study was approved by the Ethics Committee of the Sichuan Provincial People's Hospital. All patients gave fully informed written consent.

\section{Consent for publication}

Not applicable.

\section{Availability of data and materials}

The datasets used and analysed during the current study are available from the corresponding author on reasonable request.

\section{Competing interests}

The authors declare that they have no conflict of interests.

\section{Funding}

This work was supported by This work was supported by under Grant from the University of Electronic Science and Technology of China Central University Research Fund囚ZYGX2019J104囚, under Grant from the Science and Technology Project of Sichuan Province (2020YJ0447), under Grant from National Natural Science Foundation of China (No. 81970641) and under Grant from Renal Department and Institute of Nephrology, Sichuan Provincial People's Hospital, University of Electronic Science and Technology of China, Sichuan Clinical Research Center for Kidney Diseases.Dr Amanda Y Wang is supported by National Heart Foundation Post-doctoral Fellowship.

\section{Authors' contributions}

All authors have read and approved the manuscript for submission. Data collection (Shulei Fan, Qiuxia Wang ,Wei Wang, Ping Zhang, Xiang Zhong), study design (Wei Wang, Guisen Li, Li Wang), statistical analyses (Shulei Fan, Shasha Chen, Wei Wang), writing (Shulei Fan $\varangle$ Wei Wang), language modification (Wang AY).

\section{Acknowledgement}

We are grateful to all the subjects and healthy volunteers who participated in this work. Dr Amanda Y Wang is supported by National Heart Foundation Post-doctoral Fellowship.

\section{References}


1. Ronco P, Debiec H: Pathophysiological advances in membranous nephropathy: time for a shift in patient's care. Lancet 2015, 385(9981):1983-1992.

2. Ponticelli C, Glassock RJ: Glomerular diseases: membranous nephropathy-a modern view. Clinical journal of the American Society of Nephrology : CJASN 2014, 9(3):609-616.

3. Xu X, Wang G, Chen N, Lu T, Nie S, Xu G, Zhang P, Luo Y, Wang Y, Wang X et al: Long-Term Exposure to Air Pollution and Increased Risk of Membranous Nephropathy in China. Journal of the American Society of Nephrology : JASN2016, 27(12):3739-3746.

4. Xie J, Chen N: Primary glomerulonephritis in mainland China: an overview. Contributions to nephrology 2013, 181:1-11.

5. Couser WG, Steinmuller DR, Stilmant MM, Salant DJ, Lowenstein LM: Experimental glomerulonephritis in the isolated perfused rat kidney. The Journal of clinical investigation 1978, 62(6):1275-1287.

6. Van Damme BJ, Fleuren GJ, Bakker WW, Vernier RL, Hoedemaeker PJ: Experimental glomerulonephritis in the rat induced by antibodies directed against tubular antigens. V. Fixed glomerular antigens in the pathogenesis of heterologous immune complex glomerulonephritis. Laboratory investigation; a journal of technical methods and pathology 1978, 38(4):502-510.

7. Beck LH, Jr., Bonegio RG, Lambeau G, Beck DM, Powell DW, Cummins TD, Klein JB, Salant DJ: M-type phospholipase $\mathrm{A} 2$ receptor as target antigen in idiopathic membranous nephropathy. The New England journal of medicine 2009, 361(1):11-21.

8. Hofstra JM, Debiec H, Short CD, Pelle T, Kleta R, Mathieson PW, Ronco P, Brenchley PE, Wetzels JF: Antiphospholipase $\mathrm{A} 2$ receptor antibody titer and subclass in idiopathic membranous nephropathy. Journal of the American Society of Nephrology : JASN2012, 23(10):1735-1743.

9. Kanigicherla D, Gummadova J, McKenzie EA, Roberts SA, Harris S, Nikam M, Poulton K, McWilliam L, Short CD, Venning $M$ et al: Anti-PLA2R antibodies measured by ELISA predict long-term outcome in a prevalent population of patients with idiopathic membranous nephropathy. Kidney international 2013, 83(5):940-948.

10. Cattran D: Management of membranous nephropathy: when and what for treatment. Journal of the American Society of Nephrology : JASN 2005, 16(5):1188-1194.

11. Beck LH, Jr., Fervenza FC, Beck DM, Bonegio RG, Malik FA, Erickson SB, Cosio FG, Cattran DC, Salant DJ: Rituximab-induced depletion of anti-PLA2R autoantibodies predicts response in membranous nephropathy. Journal of the American Society of Nephrology : JASN 2011, 22(8):1543-1550.

12. Stanescu HC, Arcos-Burgos M, Medlar A, Bockenhauer D, Kottgen A, Dragomirescu L, Voinescu C, Patel N, Pearce $K$, Hubank $M$ et al: Risk HLA-DQA1 and PLA(2)R1 alleles in idiopathic membranous nephropathy. The New England journal of medicine 2011, 364(7):616-626.

13. Kim S, Chin HJ, Na KY, Kim S, Oh J, Chung W, Noh JW, Lee YK, Cho JT, Lee EK et al: Single nucleotide polymorphisms in the phospholipase $\mathrm{A} 2$ receptor gene are associated with genetic susceptibility to idiopathic membranous nephropathy. Nephron Clinical practice 2011, 117(3):c253-258. 
14. Liu YH, Chen CH, Chen SY, Lin YJ, Liao WL, Tsai CH, Wan L, Tsai FJ: Association of phospholipase A2 receptor 1 polymorphisms with idiopathic membranous nephropathy in Chinese patients in Taiwan. Journal of biomedical science $2010,17: 81$.

15. Lv J, Hou W, Zhou X, Liu G, Zhou F, Zhao N, Hou P, Zhao M, Zhang H: Interaction between PLA2R and HLA-DQA1 variants associates with anti-PLA2R antibodies and membranous nephropathy. Journal of the American Society of Nephrology : JASN 2013, 24(8):1323-1329.

16. Coenen MJ, Hofstra JM, Debiec H, Stanescu HC, Medlar AJ, Stengel B, Boland-Auge A, Groothuismink $\mathrm{JM}$, Bockenhauer D, Powis SH et al: Phospholipase A2 receptor (PLA2R) sequence variants in idiopathic membranous nephropathy. Journal of the American Society of Nephrology : JASN 2013, 24(4):677-683.

17. Bullich G, Ballarin J, Oliver A, Ayasreh N, Silva I, Santin S, Diaz-Encarnacion MM, Torra R, Ars E: HLADQA1 and PLA2R polymorphisms and risk of idiopathic membranous nephropathy. Clinical journal of the American Society of Nephrology : CJASN 2014, 9(2):335-343.

18. Latt KZ, Honda K, Thiri M, Hitomi Y, Omae Y, Sawai H, Kawai Y, Teraguchi S, Ueno K, Nagasaki M et al: Identification of a two-SNP PLA2R Haplotype and HLA-DRB1 Alleles as Primary Risk Associations in Idiopathic Membranous Nephropathy. Scientific reports 2018, 8(1):15576.

19. Wang W, Fan S, Li G, Wang AY, Hong D, Zhong X, Wang L: Interaction between PLA2R and HLA-DQA1 Variants contributes to the increased genetic susceptibility to membranous nephropathy in Western China. Nephrology 2018.

20. Wang HY, Cui Z, Xie LJ, Zhang LJ, Pei ZY, Chen FJ, Qu Z, Huang J, Zhang YM, Wang X et al: HLA class II alleles differing by a single amino acid associate with clinical phenotype and outcome in patients with primary membranous nephropathy. Kidney international 2018, 94(5):974-982.

21. Polanco N, Gutierrez E, Covarsi A, Ariza F, Carreno A, Vigil A, Baltar J, Fernandez-Fresnedo G, Martin C, Pons $S$ et al: Spontaneous remission of nephrotic syndrome in idiopathic membranous nephropathy. Journal of the American Society of Nephrology: JASN2010, 21(4):697-704.

22. Glassock RJ: Diagnosis and natural course of membranous nephropathy. Seminars in nephrology 2003, 23(4):324-332.

23. Cattran DC, Reich HN, Beanlands HJ, Miller JA, Scholey JW, Troyanov S, Genes G, Glomerulonephritis G: The impact of sex in primary glomerulonephritis. Nephrology, dialysis, transplantation : official publication of the European Dialysis and Transplant Association - European Renal Association 2008, 23(7):2247-2253.

24. Ramachandran R, Kumar V, Kumar A, Yadav AK, Nada R, Kumar H, Kumar V, Rathi M, Kohli HS, Gupta $\mathrm{KL}$ et al: PLA2R antibodies, glomerular PLA2R deposits and variations in PLA2R and HLA-DQA1 genes in primary membranous nephropathy in South Asians. Nephrology, dialysis, transplantation : official publication of the European Dialysis and Transplant Association - European Renal Association 2016, 31(9):1486-1493.

25. Saeed M, Beggs ML, Walker PD, Larsen CP: PLA2R-associated membranous glomerulopathy is modulated by common variants in PLA2R and HLA-DQA1 genes. Genes and immunity 2014, 
15(8):556-561.

26. Kaga H, Komatsuda A, Omokawa A, Okuyama S, Mori K, Wakui H, Takahashi N: Analysis of PLA2R and HLA-DQA1 sequence variants in Japanese patients with idiopathic and secondary membranous nephropathy. Clinical and experimental nephrology 2018, 22(2):275-282.

27. Cui G, Zhang L, Xu Y, Cianflone K, Ding H, Wang DW: Development of a high resolution melting method for genotyping of risk HLA-DQA1 and PLA2R alleles and ethnic distribution of these risk alleles. Gene 2013, 514(2):125-130.

28. De Vriese AS, Glassock RJ, Nath KA, Sethi S, Fervenza FC: A Proposal for a Serology-Based Approach to Membranous Nephropathy. Journal of the American Society of Nephrology : JASN 2017, 28(2):421430.

29. Svobodova B, Honsova E, Ronco P, Tesar V, Debiec H: Kidney biopsy is a sensitive tool for retrospective diagnosis of PLA2R-related membranous nephropathy. Nephrology, dialysis, transplantation : official publication of the European Dialysis and Transplant Association - European Renal Association 2013, 28(7):1839-1844.

30. Hoxha E, Harendza S, Pinnschmidt H, Panzer U, Stahl RA: M-type phospholipase A2 receptor autoantibodies and renal function in patients with primary membranous nephropathy. Clinical journal of the American Society of Nephrology : CJASN 2014, 9(11):1883-1890.

31. Pourcine F, Dahan K, Mihout F, Cachanado M, Brocheriou I, Debiec H, Ronco P: Prognostic value of PLA2R autoimmunity detected by measurement of anti-PLA2R antibodies combined with detection of PLA2R antigen in membranous nephropathy: A singlecentre study over 14 years. PloS one 2017, 12(3):e0173201.

32. Ma YC, Zuo L, Chen JH, Luo Q, Yu XQ, Li Y, Xu JS, Huang SM, Wang LN, Huang W et al: Modified glomerular filtration rate estimating equation for Chinese patients with chronic kidney disease. Journal of the American Society of Nephrology: JASN2006, 17(10):2937-2944.

33. Inker LA, Lambers Heerspink HJ, Mondal H, Schmid CH, Tighiouart H, Noubary F, Coresh J, Greene T, Levey AS: GFR decline as an alternative end point to kidney failure in clinical trials: a meta-analysis of treatment effects from 37 randomized trials. American journal of kidney diseases : the official journal of the National Kidney Foundation 2014, 64(6):848-859.

\section{Tables}

Table 1 Baseline clinical characteristics of the study cohort 


\begin{tabular}{|ll|}
\hline Characteristic & Patients with PMN (n=314) \\
\hline Male/female & $166 / 148$ \\
\hline Age at biopsy (year) & $49.1 \pm 13.5$ \\
\hline Male $(\mathrm{n}, \%)$ & $166(52.9)$ \\
\hline Scr $(\mu \mathrm{mol} / \mathrm{L})$ & $68.60 \pm 26.80$ \\
\hline Urea $(\mathrm{mmol} / \mathrm{L})$ & $5.80 \pm 4.11$ \\
\hline eGFR $\left(\mathrm{ml} /\left(\mathrm{min} \cdot 1.73 \mathrm{~m}^{2}\right)\right)$ & $101.33 \pm 22.67$ \\
\hline Urine protein $(\mathrm{g} / 24 \mathrm{~h})$ & $4.03(2.34,6.20)$ \\
\hline HGB $(\mathrm{g} / \mathrm{L})$ & $131.18 \pm 19.78$ \\
\hline Alb $(\mathrm{g} / \mathrm{L})$ & $27.20 \pm 15.84$ \\
\hline SBP $(\mathrm{mmHg})$ & $128.06 \pm 15.83$ \\
\hline DBP $(\mathrm{mmHg})$ & $78.23 \pm 11.24$ \\
\hline Hypertension $(\mathrm{n}, \%)$ & $78(24.8)$ \\
\hline
\end{tabular}

Table 2 Baseline and follow-up clinical characteristics of the study cohort 


\begin{tabular}{|c|c|}
\hline Characteristic & Patients with PMN $(n=186)$ \\
\hline \multicolumn{2}{|l|}{ Baseline } \\
\hline Age at biopsy (years) & $49.1 \pm 13.4$ \\
\hline Male (n, \%) & $102(54.8)$ \\
\hline $\mathrm{SCr}(\mu \mathrm{mol} / \mathrm{L} \mathbb{Z}$ & $68.9 \pm 22.5$ \\
\hline eGFR (ml/min/1.73囚) & $100.8 \pm 20.7$ \\
\hline Urea (mmol/L) & $5.6 \pm 2.2$ \\
\hline Alb (g/L】 & $28.4 \pm 19.1 \llbracket 27.6(22.2,31.4)$ \\
\hline $\mathrm{UA}(\mu \mathrm{mol} / \mathrm{L} \otimes$ & $362.0 \pm 95.5$ \\
\hline 24h-u-pro (g/d) & $4.17(2.31,7.34)$ \\
\hline $\mathrm{SBP}(\mathrm{mmHg})$ & $128.4 \pm 15.2$ \\
\hline $\mathrm{DBP}(\mathrm{mmHg})$ & $78.5 \pm 11.0$ \\
\hline Hypertension (n, \%) & $47(25.3)$ \\
\hline \multicolumn{2}{|l|}{ Follow-up } \\
\hline $\mathrm{SCr}(\mu \mathrm{mol} / \mathrm{L} \nabla$ & $92.4 \pm 89.1$ \\
\hline eGFR (ml/min/1.73囚) & $90.6 \pm 29.9$ \\
\hline Urea (mmol/L) & $6.6 \pm 3.2$ \\
\hline Alb (g/LD & $36.4 \pm 8.5$ \\
\hline $\mathrm{UA}(\mu \mathrm{mol} / \mathrm{L} \rrbracket$ & $373.3 .0 \pm 106.6$ \\
\hline 24h-u-pro (g/d) & $0.86(0.15,2.65)$ \\
\hline Follow-up time (month) & $18.6(6.7,45.5)$ \\
\hline Endpoint event $(n, \%)$ & $41(22.0)$ \\
\hline \multicolumn{2}{|l|}{ Therapy } \\
\hline Corticosteroid monotherapy(n,\%) & $29(15.6)$ \\
\hline Immunosuppression(n,\%) & $112(60.2)$ \\
\hline Non- Immunosuppression(n,\%) & $45(24.2)$ \\
\hline \multicolumn{2}{|l|}{ Treatment effect } \\
\hline \multicolumn{2}{|l|}{ Corticosteroid monotherapy } \\
\hline CR/PR/non-SR(n,\%) & $12 / 6 / 11(41.4,20.7,37.9)$ \\
\hline
\end{tabular}




\begin{tabular}{|cc|}
\hline Immunosuppression & \\
\hline CR/PR/non-SR(n,\%) & $37 / 21 / 54(33.0,18.8,48.2)$ \\
\hline Non- Immunosuppression & \\
\hline CR/PR/non-SR(n,\%) & $14 / 13 / 18(31.1,28.9,40.0)$ \\
\hline
\end{tabular}

The Endpoint event was defined as a combination of ESRD, a 30\% reduction of eGFR from baseline and double times increasing of creatinine.

Table 3 Risk factors for no remission after treatment in PMN

\begin{tabular}{|llll|}
\hline Predictor & OR & $95 \% \mathrm{Cl}$ & $P$ \\
\hline Gender (male) & 0.760 & $(0.488-1.185)$ & 0.226 \\
\hline Age & 1.018 & $(0.995-1.040)$ & 0.121 \\
\hline eGFR (ml/min/1.73囚) & 0.984 & $(0.970-0.998)$ & 0.028 \\
\hline Urea (mmol/L) & 1.184 & $(1.030-1.361)$ & 0.017 \\
\hline Alb (g/L】 & 0.922 & $(0.880-0.966)$ & 0.001 \\
\hline UA ( $\mu \mathrm{mol} / \mathrm{L} \rrbracket$ & 0.987 & $(0.971-1.003)$ & 0.655 \\
\hline 24h-u-pro (g/d) & 1.083 & $(0.998-1.174)$ & 0.056 \\
\hline Blood pressure & 0.9047 & $(0.474-1.727)$ & 0.761 \\
\hline Anti-PLA2R-antibody positivity* & 0.376 & $(0.112-1.266)$ & 0.114 \\
\hline 2715918 A allele carrier & 1.045 & $(0.560-1.950)$ & 0.891 \\
\hline 4665143 A allele carrier & 1.340 & $(0.651-2.759)$ & 0.427 \\
\hline 2187668 A allele carrier & 0.945 & $(0.525-1.708)$ & 0.851 \\
\hline
\end{tabular}

*: Both follow-up data and the number of anti-PLA2R antibodies tested were 71.

Table 4 Logistic regression analyses between SNPs within HLA-DQA1 and PLA2R genes and primary membranous nephropathy response to immunosuppressive therapy 


\begin{tabular}{|c|c|c|c|c|c|c|c|}
\hline \multirow[t]{2}{*}{ RS\# } & & Remission & $\begin{array}{l}\text { Non- } \\
\text { Remission }\end{array}$ & \multicolumn{2}{|l|}{ Univariate } & \multicolumn{2}{|l|}{ Multivariate } \\
\hline & & $n(\%)$ & $n(\%)$ & $\mathrm{OR}(95 \% \mathrm{Cl})$ & $P$ & $\mathrm{OR}(95 \% \mathrm{Cl})$ & $P$ \\
\hline \multirow[t]{3}{*}{ rs2715918 } & GG & $39(67.3)$ & $38(70.4)$ & 1 & & & \\
\hline & $\mathrm{GA}$ & 17(29.3) & $13(24.0)$ & $\begin{array}{l}0.864(0.388- \\
1.926)\end{array}$ & 0.721 & $\begin{array}{l}1.273(0.545- \\
2.978)\end{array}$ & 0.577 \\
\hline & AA & $2(3.4)$ & $3(5.6)$ & & & & \\
\hline \multirow[t]{3}{*}{ rs4665143 } & GG & $15(25.9)$ & $9(16.7)$ & 1 & & & \\
\hline & GA & $20(34.5$ & $26(48.1)$ & $\begin{array}{l}1.744(0.691- \\
4.403)\end{array}$ & 0.239 & $\begin{array}{l}2.434(0.870- \\
6.809)\end{array}$ & 0.090 \\
\hline & AA & 23(39.6) & $19(35.2)$ & & & & \\
\hline \multirow[t]{3}{*}{ rs2187668 } & GG & $34(58.6)$ & $31(57.4)$ & 1 & & & \\
\hline & GA & 23(39.7) & $22(40.7)$ & $\begin{array}{l}1.051(0.496- \\
2.227)\end{array}$ & 0.897 & $\begin{array}{l}1.139(0.521- \\
2.494)\end{array}$ & 0.744 \\
\hline & $\mathrm{AA}$ & $1(1.7)$ & $1(1.9)$ & & & & \\
\hline
\end{tabular}

aUnivariate analysis considering a dominant model for HLA-DQA1 and a dominant model for PLA2R. The non-risk genotypes for primary membranous nephropathy susceptibility were considered as the reference. ${ }^{\mathrm{b}}$ Adjusted for proteinuria at diagnosis.

Table 5 clinical characteristics of PMN patients who detected circulating anti-PLA2R antibodies

\begin{tabular}{|llll|}
\hline Characteristic & anti-PLA2R(+) & anti-PLA2R(-) & $P$ \\
\hline Baseline & $\mathrm{n}=91$ & $\mathrm{n}=29$ & \\
\hline Age at biopsy (years) & $51.07 \pm 12.66$ & $48.0 \pm 15.63$ & 0.172 \\
\hline Male $(\mathrm{n}, \%)$ & $57(62.2)$ & $15(51.7)$ & 0.296 \\
\hline Cr $(\mu \mathrm{mol} / \mathrm{L} \rrbracket$ & $73.17 \pm 25.05$ & $75.60 \pm 52.47$ & 0.736 \\
\hline eGFR $(\mathrm{ml} / \mathrm{min} / 1.73 \rrbracket)$ & $95.77 \pm 21.78$ & $100.84 \pm 27.78$ & 0.311 \\
\hline Urea $(\mathrm{mmol} / \mathrm{L})$ & $6.17 \pm 4.32$ & $5.55 \pm 2.71$ & 0.472 \\
\hline Alb $(\mathrm{g} / \mathrm{L} \rrbracket$ & $27.97 \pm 26.29$ & $27.7 \pm 7.64$ & 0.959 \\
\hline 24h-u-pro (g/d) & $5.08 \pm 3.55$ & $4.36 \pm 5.75$ & 0.436 \\
\hline SBP $(\mathrm{mmHg})$ & $131.96 \pm 15.16$ & $127.89 \pm 15.77$ & 0.222 \\
\hline DBP $(\mathrm{mmHg})$ & $79.55 \pm 10.68$ & $78.39 \pm 10.22$ & 0.614 \\
\hline
\end{tabular}


Table 6 Baseline characteristics of 120 PMN patients for detecting circulating anti-PLA2R antibody

\begin{tabular}{|llllll|}
\hline & $\begin{array}{l}\text { PLA2R low } \\
\text { risk+ HLA low } \\
\text { risk }\end{array}$ & $\begin{array}{l}\text { PLA2R low } \\
\text { risk+ HLA } \\
\text { high risk }\end{array}$ & $\begin{array}{l}\text { PLA2R high } \\
\text { risk+ HLA low } \\
\text { risk }\end{array}$ & $\begin{array}{l}\text { PLA2R high } \\
\text { risk+ HLA } \\
\text { high risk }\end{array}$ & $P$ \\
\hline Male/female & $8 / 4$ & $\mathrm{n}=7$ & $\mathrm{n}=60$ & $\mathrm{n}=41$ & \\
\hline Age(year) & $48.33 \pm 15.47$ & $54.86 \pm 15.08$ & $49.95 \pm 15.28$ & $52.71 \pm 9.30$ & 0.564 \\
\hline Scr( $\mu \mathrm{mol} / \mathrm{L})$ & $79.75 \pm 28.70$ & $73.78 \pm 22.95$ & $72.87 \pm 39.41$ & $73.30 \pm 27.08$ & 0.935 \\
\hline Urea(mmol/L) & $5.78 \pm 1.90$ & $6.08 \pm 2.74$ & $6.43 \pm 5.19$ & $5.48 \pm 2.21$ & 0.707 \\
\hline eGFR(ml/(min-1.73m2)) & $94.49 \pm 27.64$ & $91.90 \pm 27.57$ & $99.98 \pm 23.42$ & $94.26 \pm 21.48$ & 0.577 \\
\hline Urine protein (g/24h) & $8.30 \pm 7.67$ & $3.30 \pm 2.07$ & $4.73 \pm 3.68$ & $4.38 \pm 3.09$ & 0.010 \\
\hline HGB (g/L) & $132.25 \pm 17.11$ & $132.14 \pm 15.66$ & $132.08 \pm 15.72$ & $133.07 \pm 17.52$ & 0.993 \\
\hline Alb(g/L) & $23.11 \pm 5.67$ & $28.44 \pm 7.23$ & $25.74 \pm 5.89$ & $32.40 \pm 38.65$ & 0.465 \\
\hline anti-PLA2R(+)/(-) & $5 / 7$ & $5 / 2$ & $45 / 15$ & $36 / 5$ & 0.012 \\
\hline anti-PLA2R(+)(\%) & 41.6 & 71.4 & 75.0 & 87.8 & \\
\hline
\end{tabular}

\section{Figures}




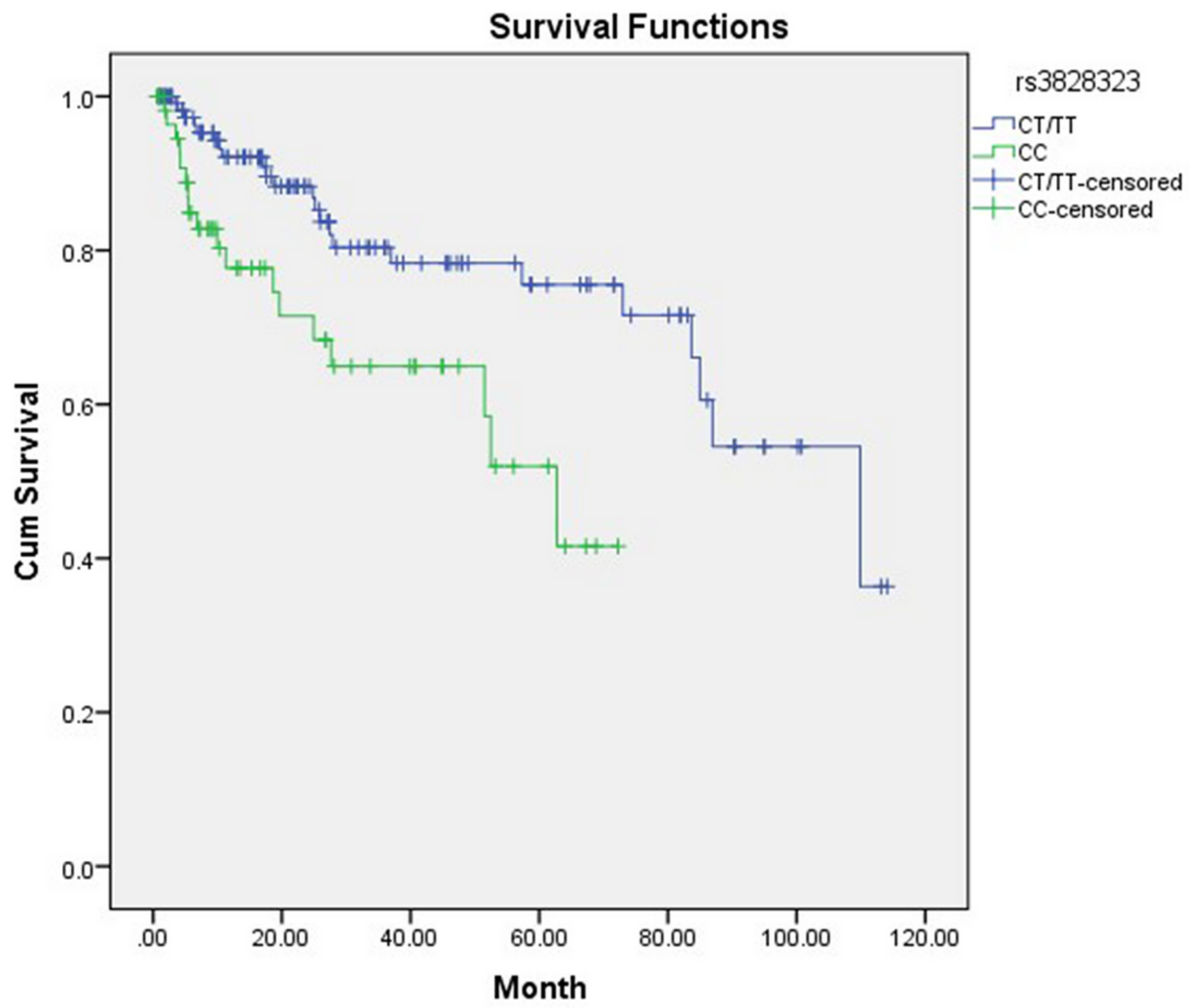

Figure 1

Survival analysis of time with outcome Kaplan-Meier analysis showed that with the prolongation of follow-up time, the CT/TT genotype for rs3828323 achieved higher cumulative survival rate $(P=0.003)$ than CC genotype.

\section{Supplementary Files}

This is a list of supplementary files associated with this preprint. Click to download.

- SupplementaryTable.docx 